\title{
Fusion of Radar and EO-sensors for Surveillance
}

\author{
Leon J.H.M. Kester, Ame Theil \\ TNO Physics and Electronics Laboratory, \\ P.O. Box 96864, 2509 JG The Hague, \\ The Netherlands
}

\begin{abstract}
Fusion of radar and EO-sensors is investigated for the purpose of surveillance in littoral waters is. All sensors are considered to be co-located with respect to the distance, typically 1 to $10 \mathrm{~km}$, of the area under surveillance. The sensor suite is a coherent polarimetric radar in combination with a set of camera's sensitive to visible light, near infrared, mid infrared and far infrared. Although co-located, the sensors are dissimilar and not necessarily synchronized. A critical aspect for beneficial fusion in this application is correct association of information from these sensors. Various architectures are considered and it will be argued that a fuse while track algorithm is the most suitable algorithm in this case. Discussed is how such an algorithm is designed and applied. To improve association reliability also non-kinematic features of both sensor types are considered. Investigated in particular is, which features from contacts measured with the polarimetric radar and the EOsensors are correlated. These features and their correlations are incorporated in the tracking process. Preliminary results are shown.
\end{abstract}

Keywords: Sensor fusion, tracking, data association, surveillance.

\section{INTRODUCTION}

The object of the study is to improve surveillance capabilities in littoral waters using a coherent polarimetric radar and a dedicated EO-sensor suite. The typical distance up to which these capabilities should be provided is 1 to $10 \mathrm{~km}$. For these ranges both radar and EO-sensors perform relatively well. However, since both types of sensors are dissimilar, fusing their data into one system could potentially yield a reduction of the number of false tracks and improved classification capabilities.

\section{FUSING RADAR AND EO-SENSORS}

\subsection{Introduction}

Aspects that have to be addressed for sensor fusion are:

1. Data association

Which data from one sensor can be combined with data from another sensor?

2. Data fusion

How should the data be combined?

3. Sensor management

How should the system be managed such that sensors and algorithms are used optimally?

Although situation assessment is also an important aspect of sensor fusion, the end result that is currently aimed at is a recognized surface picture. This result can subsequently be used to perform situation assessment, either by an operator or by a following algorithm.

The main reason for fusion of radar and EO-sensors is to exploit the complementary data of both types of sensors. However. to exploit the complementary data, the association of this data from both sensors should be unambiguous. Therefore, the best performance is achieved if there is a balance between complementary data that provides more information about the environment and data common to both types of sensors to ensure that the information is from the same physical entity. 


\subsection{Data association}

To get a better understanding of the problems which arise from the association of data of radar and EO-sensors, figure 1 shows a scene observed by a camera while figure 2 shows a range-Doppler diagram obtained with a coherent radar looking in the same direction as the camera.

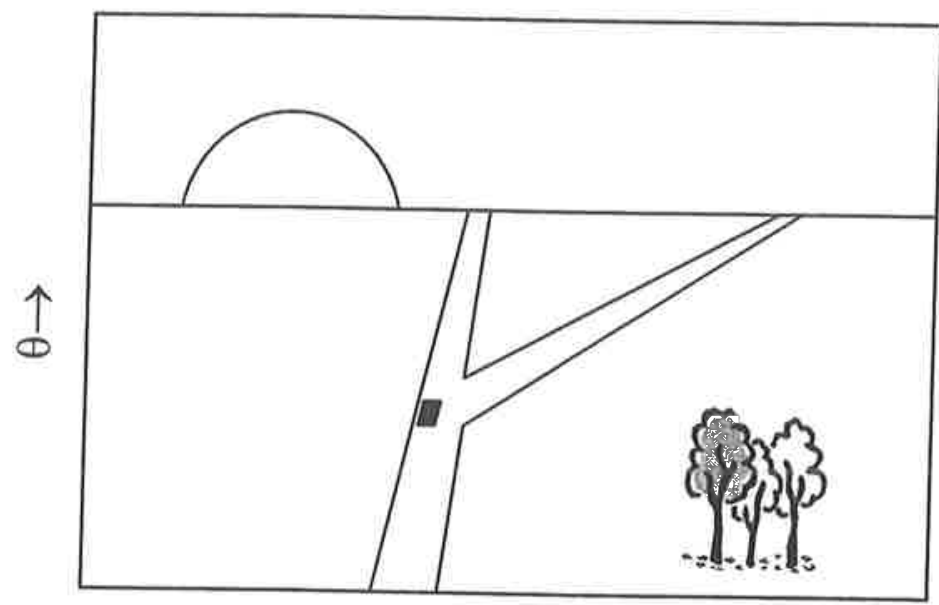

$$
\varphi \rightarrow
$$

Figure 1: Picture of an object at a specific elevation and azimuth.

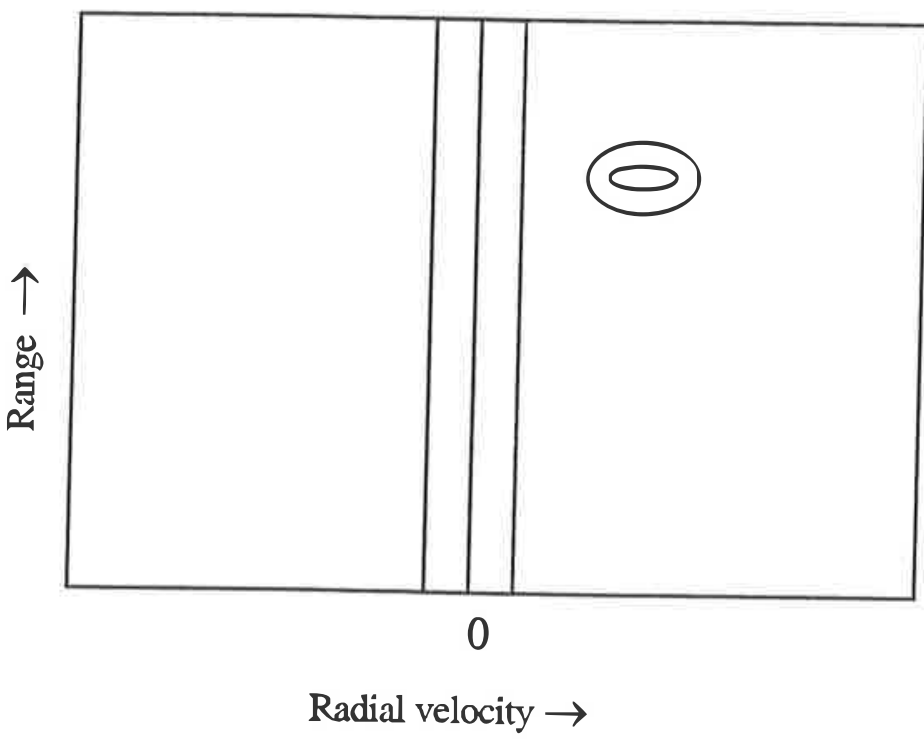

Figure 2: Range-Doppler diagram measured by a coherent radar. An object is moving with a specific radial velocity at a specific range.

The camera accurately measures elevation and azimuth; the radar however measures range and radial velocity by means of the Doppler frequency shift. This example illustrates the problem of associating data from radar and EO-sensors; there are no primary features that can be used to associate data from both types of sensors. The situation is not as bad as it seems though, because;

- From a series of pictures from the camera, objects that are moving in azimuth and elevation can be detected. Objects moving in azimuth or elevation most likely also move in range. If there are many moving objects, however, ambiguity
still exists.

- Most radars designed for surveillance can determine target azimuths fairly well.

- For surface bound objects, the elevation measured by the camera is not independent of the range measured by the radar. The same holds of course for change in elevation and radial velocity. The correlation is weakened, however, if sea waves are large and unpredictable. 
- Since both the EO-systems and the radar are sophisticated sensors, features from both data streams that correlate could be extracted. Similar to the correlation between elevation and range, such a correlation can help to reduce the uncertainties in data association.

\subsection{Data fusion}

Once data association has taken place, the data should be combined. It is important to note that the data used for data association, which is referred to as fusion features, is not necessarily the same as the data that is subsequently fused. This is because the product of the fusion process should be data that is optimal for the next process.

\subsection{Sensor management}

Besides the obvious advantage of combining data of both sensor types to improve detection, tracking and classification, information of the sensors could also be used to manage the system. Since the sensor system will be designed to operate on a ship, the sensors must be co-located. It is however quite conceivable that the sensors do not operate synchronously. The radar, for example, can cue an EO-sensor, in order to inspect a specific area. In table 1 the possibilities are listed:

Table 1: Survey of combinations of modes of operation for radar and EO-systems.

\begin{tabular}{|c|c|c|c|}
\hline & \multicolumn{2}{|c|}{ Mode } & \\
\hline & Radar & EO system & Remarks \\
\hline 1 & Search & Search & Not necessarily synchronously. \\
\hline 2 & Search & Track & Radar cues EO-system \\
\hline 3 & Search & Dual Mode & For example if the EO-system has full azimuth coverage of the area under surveillance. \\
\hline 4 & Track & Search & EO-system cues the radar. \\
\hline 5 & Track & Track & This is not an option for surveillance. \\
\hline 6 & Track & Dual Mode & See no 3. \\
\hline 7 & Dual Mode & Search & E.g., a phased array radar. \\
\hline 8 & Dual Mode & Track & \\
\hline 9 & Dual Mode & Dual Mode & The most flexible and also most expensive option. \\
\hline
\end{tabular}

The radar is limiting the number of options most, since it is the more expensive sensor. For this reason, a (non-stabilized) search radar is chosen. For the same reason, a dual mode EO-system is not considered yet. Sensor management aspects therefore concentrate on the first two combinations in table 1.

\section{ARCHITECTURE}

Before we examine the problem of how sensor fusion between radar and EO-sensors can be established best, the architecture of a general signal-processing scheme for a single sensor is discussed. This scheme, common to both types of sensors, is then used to examine at what level data fusion can best be performed.

\subsection{Single sensor signal processing scheme}

In the customary signal-processing scheme, a number of processes is identified: pre-processing, detection, tracking and classification. The result of the last process is classified tracks that compose the recognized surface picture. In figure 3 the signal-processing scheme is presented.

The sequence of the processes is similar for different types of sensors. Whether all processes are applied, however, depends on the application. There may be no interest in recognizing the objects or there may not be any need to estimate the properties of the objects with a tracking process.

The reason that the tracking process is performed before the classification process is because in tracks the information of a number of contacts is integrated. This will lead to a better classification. Each track, for example. can contain the minimum and maximum velocity of the object. which excludes certain classes or the behavior of the object in time reveals features that improve classification results.

One might argue that if the classification process precedes the tracking process, classification results could improve tracking. If, for example, a contact is classified as a sailboat it can only be associated to the track of a sailboat (if there are 
no ambiguous classes). It is important to realize, however, that the task of each process is limited to reduce and transform the data in such a way that it is optimal for the next process. The process preceding the tracking process should therefore provide optimal information for the tracking process. This information may be a certain class but it is favorable to use the features on which this classification result is based. A feature 'red' measured with an EO-sensor or 'modulation frequency' measured with a radar will be more useful to the tracking process than a classification result derived from these features. This is because a classification process is usually not a one to one mapping from features to classes and therefore destroys information. It is, however, conceivable that some kind of classification is performed with contact features. This is regarded as feature extraction, which is part of the detection process.

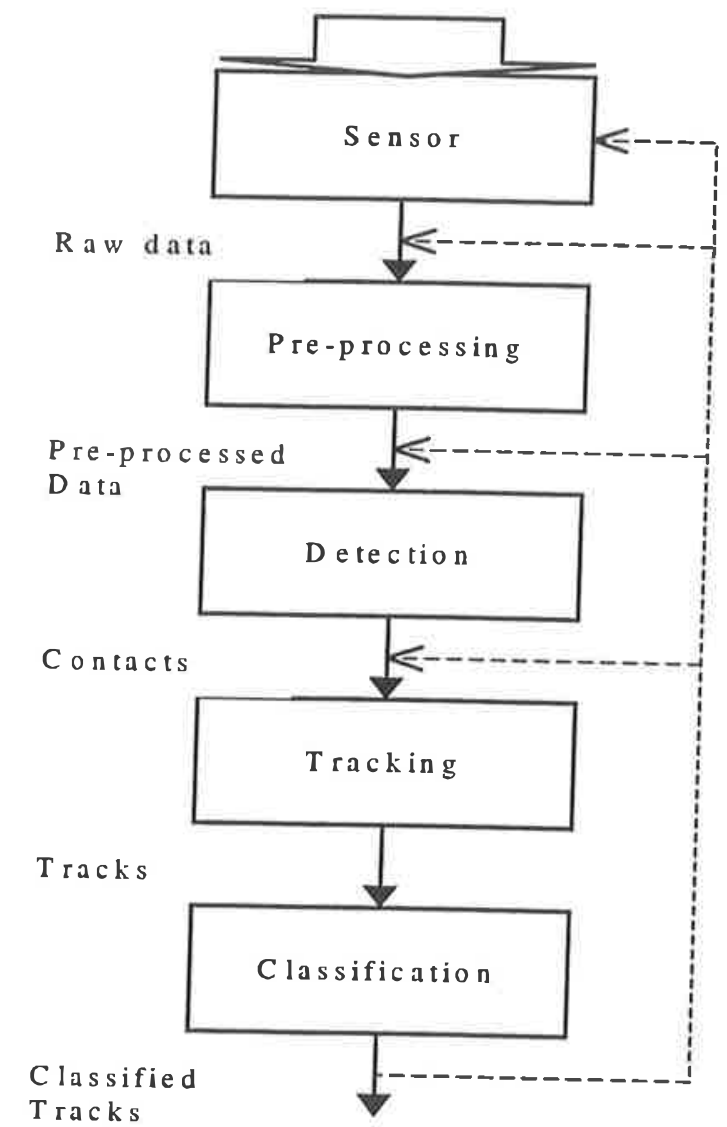

Figure 3: Scheme of the processes in the single sensor signal processing chain, including sensor and processing management.

Also shown in figure 3 is the sensor and processing management performed by feeding back the results from the processe to earlier processes and to the sensor itself. The more the system manages itself, the more intelligent the system must be since it must be aware of the implications of changing the algorithm or the sensor settings.

\subsection{Multi sensor signal processing scheme}

In the case of more than one sensor there are several data streams. The object is, however. to have a single recognized surface picture. Therefore, somewhere in the signal-processing scheme, the data streams have to be fused. In principle fusion can be performed at more than one level. In figure 4 a general signal-processing scheme is shown for more than one
sensor, thus including fusion at every level.

In the single sensor scheme processes are optional. This also applies to the fusion processes. The dotted line between a fusion process and the subsequent process indicates that both processes can not always be separated. If the fusion process can be separated, however, it precedes the other process. The gradually smaller arrows indicate the decreasing number of
independent data streams. 
Similar sensors such as camera's that are co-located can be fused at any level since already the raw or pre-processed data are represented in the same data space. For dissimilar sensors like an EO-sensor and radar, fusion of raw data does not seem feasible. Trying to associate pre-processed data of both sensors will, apart from the profit, certainly demand a large amouns of processing power. Besides that, the sensors have to operate synchronously. The demand of processing power is diminished at the detection level since the detection process reduces the amount of data to only the regions of interest and subsequently the contacts. Fusion of contacts offers the possibility to associate data using features extracted in the detection process. The features that can be extracted depend of course on how sophisticated the sensors are. It is obvious that contacts of a multi-spectral EO-system provides more information and therefore its contacts contains more features than contacts obtained with a black and white camera. Likewise, contacts obtained with a coherent polarimetric radar will contain more features than contacts obtained with a non-coherent radar. As mentioned before, for an optimal performance of the multisensor system, each sensor must be sensitive to one or more common features to be able to associate the others. Although fusion of contacts before the tracking process seems feasible and useful, there are three disadvantages. The sensors have to operate synchronously or nearly synchronously, the fusion process does not use the measurement history and the process is sensitive to sensor failure.

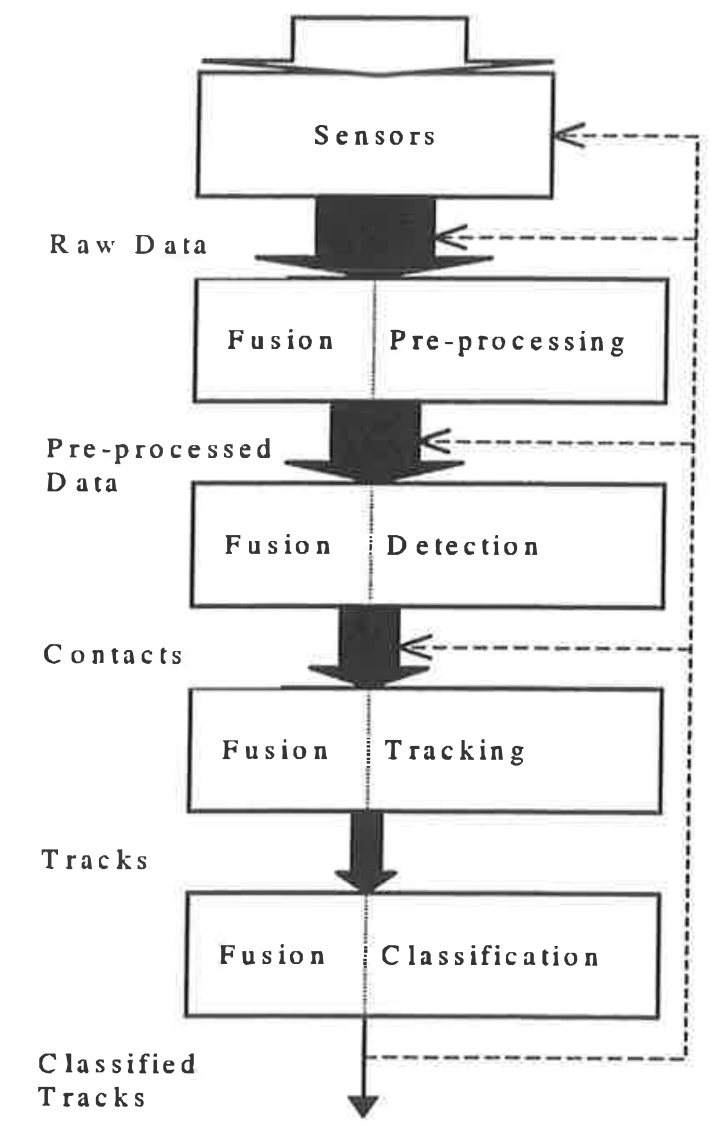

Figure 4: Scheme of the processes in the multi-sensor signal processing chain including sensor and process management.

A solution to all three problems is to integrate the fusion process in the tracking process. This process must be able to handle contacts from both sensor types which arrive asynchronously and integrate that to tracks represented in a global space. The process must be able to predict new measurements for both sensors. In this way the contacts are fused in tracks while the track history improves the association.

One step further down the chain is fusion of tracks. Although history can be used in the fusion process, the individual track processes of both types of sensors are less accurate since they were not aware of the information provided by the other sensor. Moreover, track fusion is far from trivial since it has to deal with lost, broken, mixed up, ambiguous and false tracks from both track processes. Especially for dissimilar sensors, the results of these processes can be quite different. 
It can be concluded that fusion integrated in the tracking process offers the best prospects for fusion of EO-sensors and radar. In the next paragraph we discuss how this can be implemented.

\section{FUSE WHILE TRACK}

Before examining how the fusion process can be incorporated in the tracking process, the tracking process itself is discussed.

The geometrical and dynamic features of the contacts are defined in the polar coordinate system of the sensor. The tracks however, are usually defined in the Cartesian space, which is more suitable for maneuvering objects and independent of the sensor. If there is more than one sensor and the sensors are dissimilar it is even more important that the state space is not dependent of the sensors.

Note that the measurement space and state space in which the contacts and tracks are defined are not limited to the 3-D space in which the kinematical quantities are defined. Also features like temperature. signal modulation or radar cross section can be dimensions in this space. Conditions are that transformations between both spaces are well defined and that there is a model that is able to make predictions.

A general scheme for the tracking process is shown in figure 5.

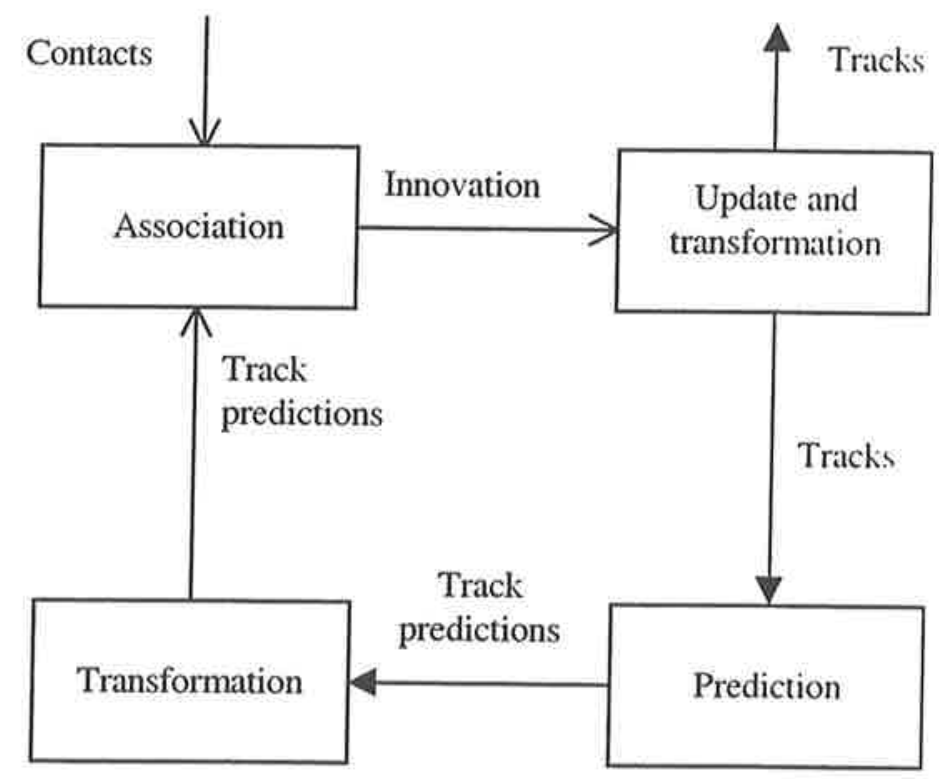

Figure 5: General scheme for the tracking process. Open arrows represent data specified in the measurement space while closed arrows represent data in the state space.

In this scheme contacts are associated to predictions from existing tracks in measurement space. The result of this association is used to update existing tracks and possibly to create new tracks in the state space. In most single sensor applications, the time interval between measurements is fixed. Therefore predictions can be made as soon as the tracks are updated. Then the predictions are transformed to the measurement space. At the next point in time, when new contacts are measured, the process starts again.

How can this tracking process be used to fuse data from dissimilar and possibly asynchronously operating sensors? In this case there are contacts defined in a sensor specific space while the results are global tracks. associated to objects in the state space. The prediction process does not have to be altered. It must however be supplied with the point in time at which the next measurement (by any of the sensors) has been performed. The transformation process must know to which measurement space the predictions have to be transformed. The scheme for this process is shown in figure 6 . 
This process is capable of fusing data of synchronously as well as asynchronously operating sensors. If they operaie synchronously, the result should of course not depend significantly on the order in which the simultaneous measurements
are processed.

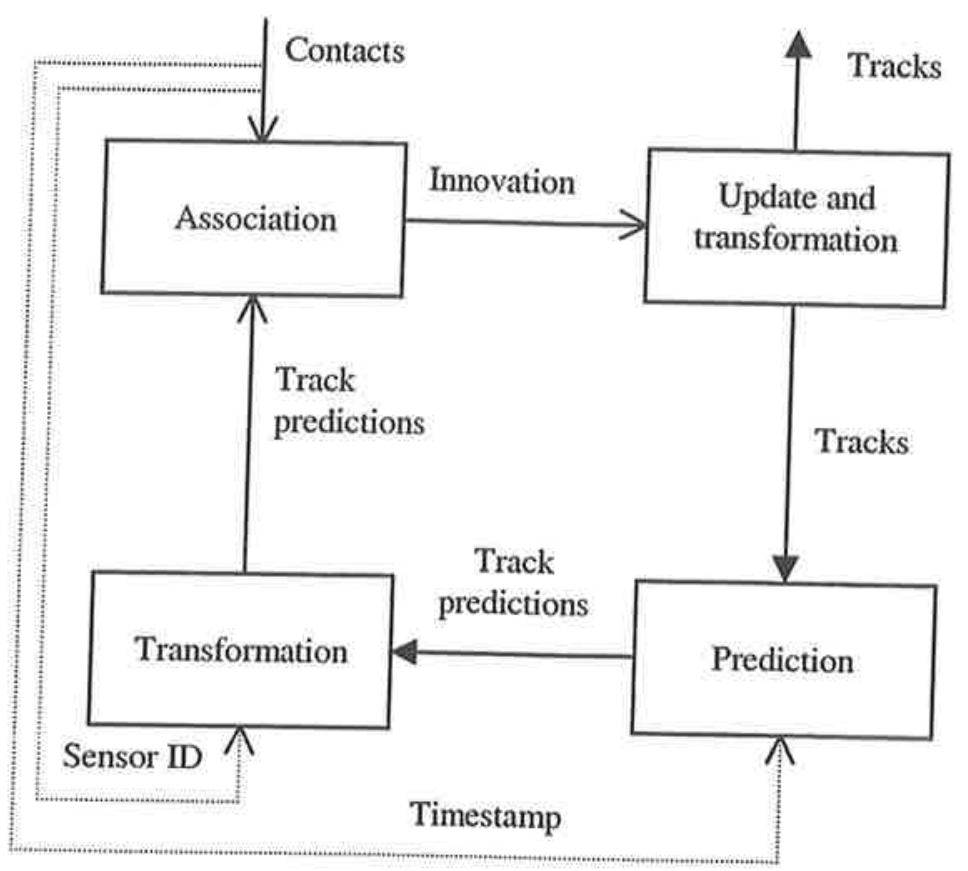

Figure 6: General scheme for the tracking process. Open arrows represent data specified in the measurement space while closed arrows represent data in the state space.

Before examining in more detail how the so-called fuse while track algorithm can be implemented, the question must be addressed what type of tracking process is most suitable. Two candidates are considered:

- Kalman filter [1]

The prediction depends only on the current state and the time interval (Markov process). It assumes a normal probability density distribution for the parameters. Process noise is introduced to allow maneuvers that are not predicted by the model. If the transformation between the space in which the contacts are defined (measurement space) and the space in which the tracks are defined (state space) is not linear, the extended Kalman filter (EKF) [2] needs to be used.

- Batch filter.

This filter can be used if predictable but non-linear maneuvers of the objects of interest are expected. The batch filter uses a limited number of previous measurements to determine the type of maneuver. The processing load for this filter is usually severe. A priori knowledge is needed about the expected type of maneuvers that are allowed.

The Kalman filter has a more general structure, which allows a more flexible implementation. Also, substantially more research has been done on the Kalman filter than on the batch filter. A better performance for maneuvering objects can be achieved by an interacting multiple model approach (IMM) [2] or by adding parameters to the state vector that are related to the behavior of the object. By means of the covariance matrix that reflects the correlation between the parameters, the prediction becomes dependent on the specific object.

In figure 7, a scheme is shown that is analogue to the scheme of figure 6 but now specific for the Kalman filter. In this scheme it is assumed that the measurement covariance matrix is independent of the measurement itself. If this is not the case, besides the sensor $\mathrm{ID}$, also the measurement covariance matrix needs to be supplied to the process that calculates the Kalman gain. In the case of the EKF also the measurement matrix that transforms track predictions from state space to measurement space needs to be supplied to the process that updates the covariance matrix. The figure, however, only shows the additional information that is needed in the asynchronous multi-sensor fuse while track algorithm. 


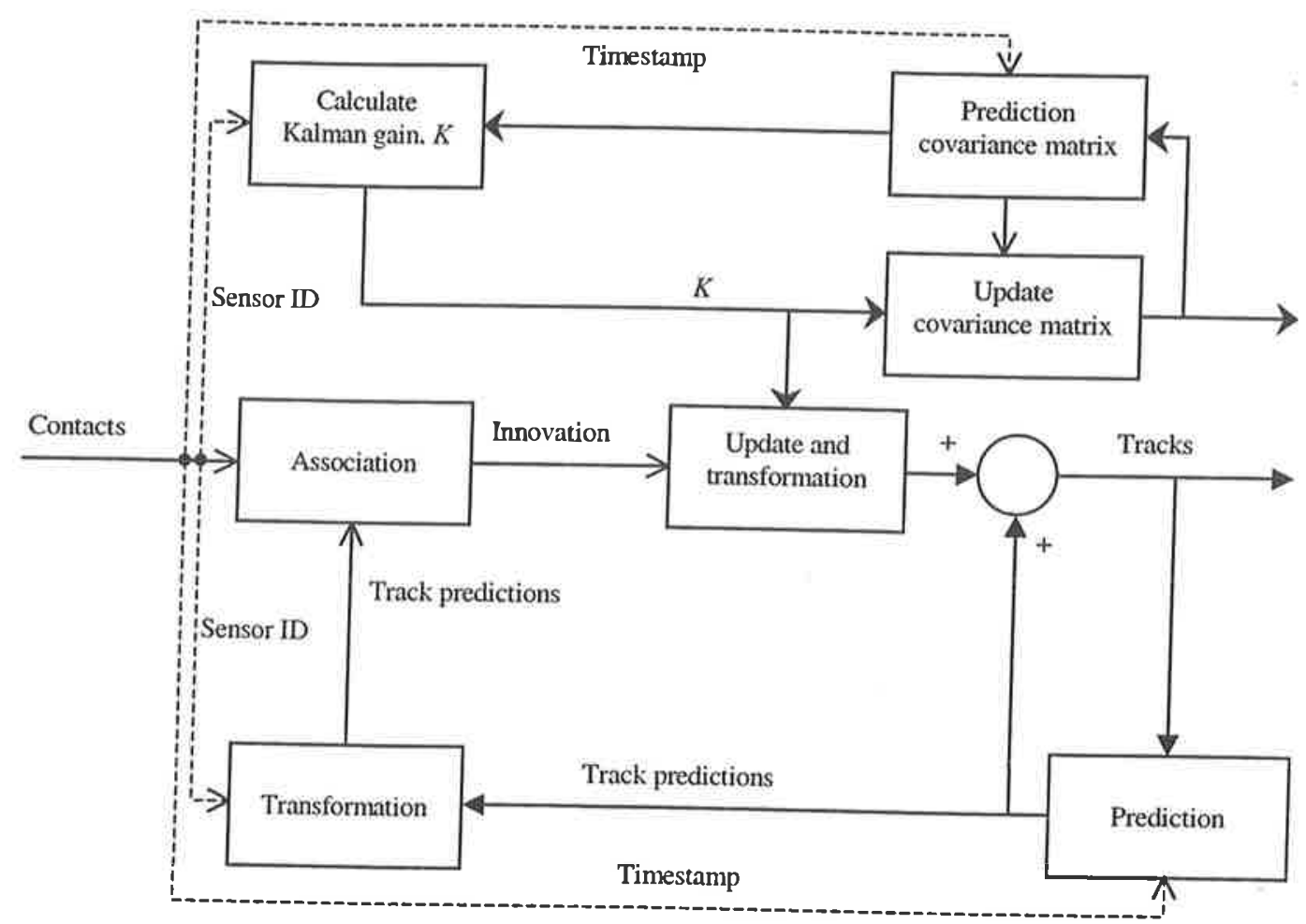

Figure 7: Scheme for the Kalman filter process with contacts originating from different and possibly asynchronously operating sensors.

As an example, figure 8 shows how such a procedure reduces the uncertainty in azimuth by fusing synthetic contacts from a 3D-surveillance radar and an IRST. The track is initiated by two successive radar contacts (crosses) after which also EOcontacts (dots) are considered for association. The figure shows that the azimuth is considerably better determined ivhen EO-contacts are included. More important however is that this process achieves the most reliable association such that (other) complimentary features can be exploited to classify the tracked object.

Another issue that needs to be addressed, is the association of contacts to predictions. Three possibilities are considered:

- Nearest Neighbor

Every contact is uniquely associated to a track such that the overall likelihood is largest.

- Joint Probability Density Association (JPDA) [2]

All contacts within the track gate are, weighted with their individual association probabilities, combined.

- Multi Hypothesis Testing or Tracking [3]

Decisions are delayed by retaining several association hypotheses. During the tracking process the number of hypotheses increases dramatically. At a certain stage the number of hypotheses has to be reduced by merging them
and/or disregarding less probable hypotheses.

The fuse while track algorithm should be able to process asynchronously arriving contacts. Since the time interval between successive measurements is variable. an MHT approach seems more suitable. This is because the shorter the time interval, the more the association problems for both measurements are correlated. The two other methods do not take this into
account. 


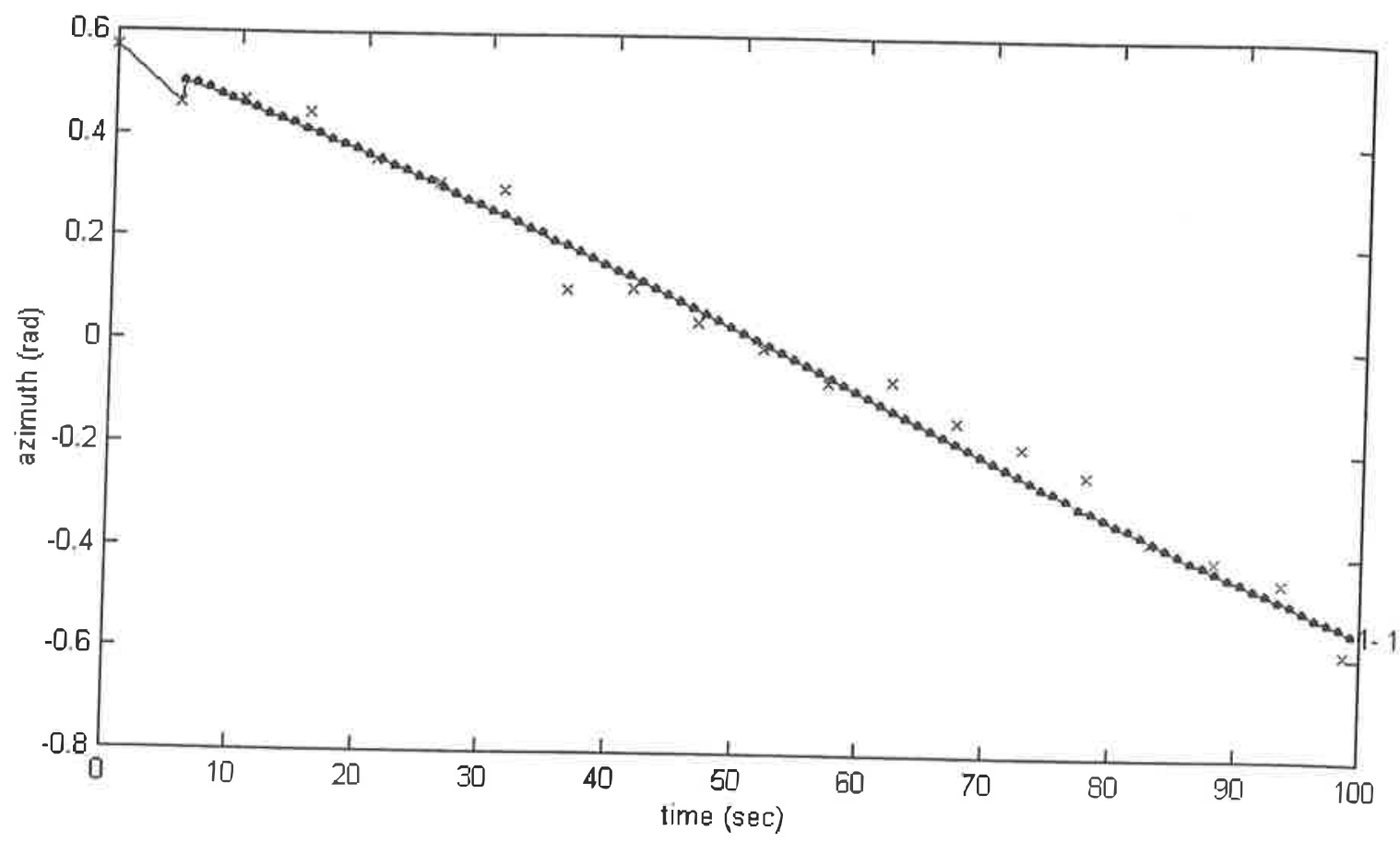

Figure 8: Azimuth of a global track (solid line) derived from radar contacts (crosses) and EO-contacts (dots).

\section{IMPLEMENTATION ASPECTS}

For these dissimilar sensors data association is a critical aspect. Research is, therefore. currenlly concentrated on identifying features from the EO-system and the radar that are correlated. As an example of such comelations. the panel on the left of figure 9 shows a typical picture of the sea surface in mid infrared. The panel on the right shows the typical difference in radial velocity between the $\mathrm{HH}$ and VV channel of sea clutter as measured with the polarimetric radar. At the corresponding azimuth and range (in the picture derived from the elevation) there is a correlation between the white caps in the picture and the difference in radial velocity.

In March 2001 a large measurement campaign has been performed that will help to char in more detail the correlations between the radar and EO features for optimal association of the contacts.
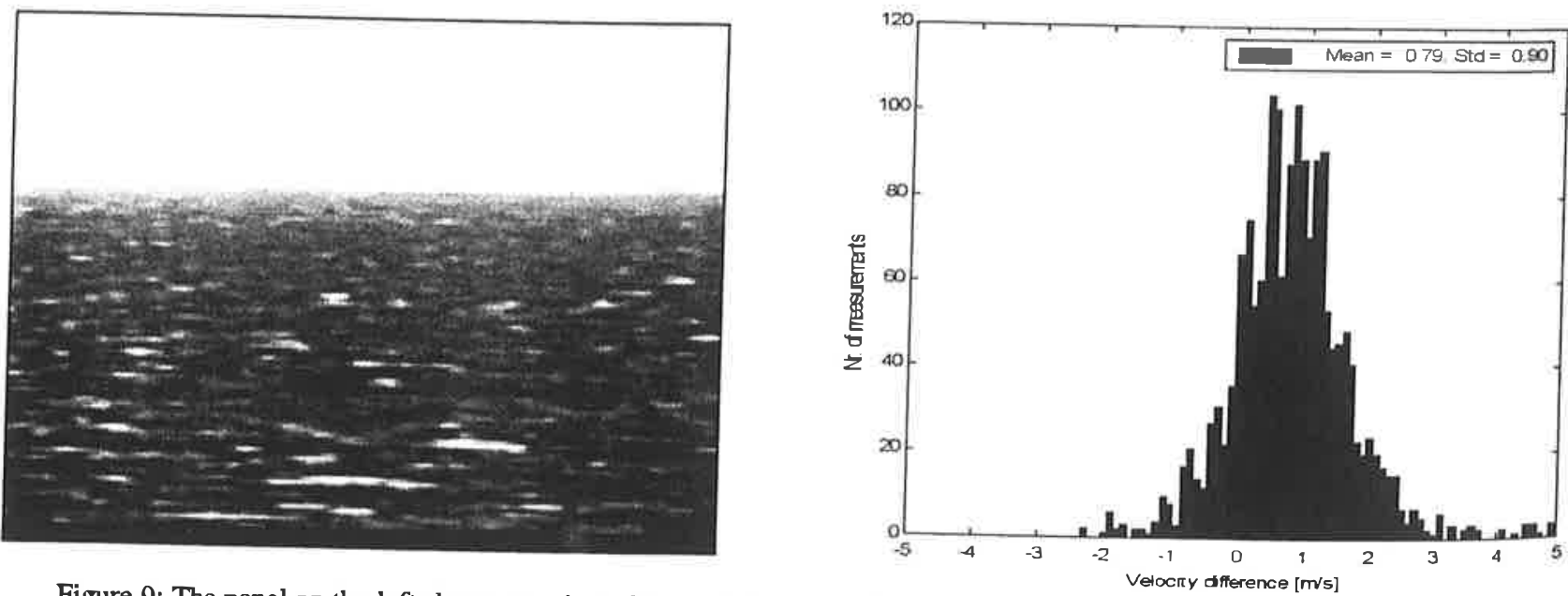

Figure 9: The panel on the left shows a typical picture of the sea surface in mid infrared. The panel on the right shows the typical difference in radial velocity between the $\mathrm{HH}$ and $\mathrm{VV}$ channel of sea clutter as measured with the polarimetric radar. 
Although the principle of MHT is simple, limiting the number of hypotheses to reasonable values, while retaining the most promising, is far from that. The best strategy depends on the performance of the detection processes and will be developed sinultaneously with those.

Adding process noise is a crude method to account for accelerations, not present in the prediction model. An IMM approach miay improve the performance whereby specific models of objects, floating or moving on the sea surface, can be implemented. Also the measured environment of the sea surface could help to predict the motion of the object.

To be able to calculate the probabilities of hypotheses, on arrival of a measured contact, the probability is needed of not detecting the object with any sensor in the time between the last and current measured contact. Accurate $a$ priori knowledge of detection probabilities in space and time are needed to estimate the probability of a missed detection. The probability that the measured contact is a false alarm might be derived from the measurements in which clutter is present.

\section{CONCLUSIONS}

In this paper fusion of EO-sensors and radar for surveillance is discussed. For possibly asynchronously operating sensors, lusion before the tracking process is not attractive since the tracking process is the optimal process for interpolation and istrapolation in time. If fusion is performed after the tracking process, the sensor specific tracking processes are not aware if the information provided by other sensors. Besides that, a complicated track to track association problem has to be colved. Most appropriate therefore is integration of the fusion into the tracking process.

This fuse while track algorithm processes contacts on arrival. Predictions and transformation of the predictions to the sensor measurements, an association algorithme delayed until a contact antives. Due to the variable time interval between successive process does not depend on the presence of retains a number of hypotheses seems most suitable. Since the fuse while track forible and not prone to sensor failure.

waters. The system consists of a coherem will be examined for a system capable of recognizing small objects in littoral mid infrared and far infrared. All sensors are co-located.

Aspects that need further investigation are: - Improvement of reliability of data association, identifying contact features of the EO-system that correlate to contact
features of the radar.

- Pruning of the MHT hypothesis tree.

- Applicability of IMM with realistic models for floating or moving objects on the sea surface.

- Incorporation of probabilities of missed detections.

- Determination of probabilities of false alarms.

\section{REFERENCES} 1. R.E.Kalman, A new approach to Linear Filtering and Prediction Problems, Trans. ASME, J. Basic Engineering. 82:34--
45, March 1960. 2. Y. Bar-Shalom and X.R. Li, Estimations and Tracking: Principles. Techniques and Software, Artech House. Boston,
1993.

3. S. S. Blackman and R. Popoli, Design and analysis of Modern Tracking Systems, Artech House, Norwood. MA. 1999. 